\title{
Estudio coste-efectividad sobre la medición del gradiente de presión venosa hepática en la profilaxis secundaria de la hemorragia digestiva varicosa
}

\author{
A. Amorós, J. M. Palazón, P. Zapater¹ , E. de Madaria y M. Pérez-Mateo \\ Unidad Hepática ${ }^{I}$ Servicio de Farmacología. Hospital General Universitario de Alicante. Universidad Miguel Hernández. \\ Alicante
}

\section{RESUMEN}

Objetivo: la hemorragia digestiva es una complicación frecuente en pacientes con cirrosis hepática. La tasa de recidiva tras un primer episodio oscila en torno al $60 \%$, motivo por el cual la profilaxis está recomendada. Esta puede realizarse mediante fármacos (betabloqueantes y nitratos) combinados o no con ligadura endoscópica con bandas. El objetivo de este estudio es valorar el coste-efectividad de la medición del gradiente de presión venosa hepática (GPVH) previo a la elección de la profilaxis secundaria.

Métodos: creamos un árbol de decisión para calcular el costeefectividad de dos estrategias: grupo 1: pacientes a los que se les determinó el GPVH; cuando tras la administración de propranolol hubo una disminución del gradiente $\geq$ al $20 \%$ respecto al inicial o disminuyó por debajo de $12 \mathrm{mmHg}$, los pacientes fueron tratados con propranolol. Si no hubo tal variación del GPVH, se realizó ligadura endoscópica de las varices. Grupo 2: en este grupo no se monitorizó el GPVH. Los pacientes con varices grado I recibieron tratamiento con betabloqueantes más nitratos y los que presentaban varices grandes (II, III, IV) fueron tratados con betabloqueantes y LEB.

Resultados: en el grupo del estudio hemodinámico respondió un $36 \%$, estos recibieron tratamiento betabloqueante, la tasa de resangrado fue del $0 \%$. En los no respondedores la tasa de resangrado fue de un $17 \%$. En el grupo sin estudio se trató con propranolol más nitratos al $28,42 \%$ y resangraron un $25 \%$; la tasa de resangrado en el grupo que recibió tratamiento con betabloqueantes más ligadura endoscópica fue de un $13 \%$. El coste total en el grupo al que se realizó el estudio hemodinámico fue de 14.100,49 euros y de 14.677,16 euros para el grupo sin estudio hemodinámico.

Conclusiones: la realización del estudio hemodinámico es una herramienta coste-efectiva en la profilaxis de la hemorragia digestiva varicosa en pacientes cirróticos y mantiene una relación coste efectiva favorable comparado con la no realización del mismo.

Palabras clave: Hemorragia digestiva varicosa. Estudio hemodinámico. Betabloqueantes.

\begin{abstract}
Objective: variceal rebleeding is common following a first episode of hemorrhage in cirrhotic patients. The objective of this study was to determine the cost-effectiveness of monitoring hepatic venous pressure gradient (HVPG) to guide secondary prophylaxis.

Methods: we created a Markov decision model to calculate cost-effectiveness for two strategies: Group 1: HVPG monitoring to decide treatment -when portal pressure was reduced by at least 20 percent or HVPG was less than $12 \mathrm{mmHg}$ after beta-blocker administration, patients received beta-blockers; when portal pressure did not meet these criteria therapy was endoscopic band ligation. Group 2: in this group there was no monitoring of HVPG. Patients with large varices received treatment with beta-blockers combined with EBL; patients with small varices received betablockers plus isosorbide mononitrate.

Results: there was no recurrent variceal bleeding in group 1 for good responders, and for $17 \%$ of poor responders. In group 2 a $25 \%$ rebleeding rate was detected in patients with small varices and $13 \%$ for those with big varices. Overall cost in group 1 was $14,100.49$ euros, and 14,677.16 in group 2 .

Conclusions: HVPG measurement is cost-effective for the secondary prophylaxis of variceal bleeding.
\end{abstract}

Key words: Variceal bleeding. Hemodynamic study. Beta-blockers.

Amorós A, Palazón JM, Zapater P, de Madaria E, Pérez-Mateo M. Estudio coste-efectividad sobre la medición del gradiente de presión venosa hepática en la profilaxis secundaria de la hemorragia digestiva varicosa. Rev Esp Enferm Dig 2008; $100: 416-422$.

\section{Recibido: 01-04-08.}

Aceptado: $29-04-08$

Correspondencia: Amparo Amorós Pina. Unidad Hepática. Hospital General Universitario. C/ Pintor Baeza, s/n. 03010 Alicante. e-mail: amparoamoros@hotmail.com

\section{INTRODUCCIÓN}

La hemorragia digestiva alta secundaria a la rotura de varices esofagogástricas es una de las principales complicaciones de la hipertensión portal. Esta acontece en el 25- 
$35 \%$ de los cirróticos y es la causa del $80-90 \%$ de los episodios hemorrágicos de estos pacientes $(1,2)$. La mortalidad de cada episodio oscila en torno al $20 \%$ y aproximadamente un $60 \%$ de los enfermos que sobreviven a un primer episodio presentará una recidiva hemorrágica sin profilaxis (3). El 30-40\% de las recidivas sucede durante las primeras seis semanas posteriores al episodio inicial. Tras la primera hemorragia, la supervivencia al año disminuye al $50 \%$ (3).

El riesgo de sangrado de las varices está condicionado por varios factores. El más importante de ellos es el aumento del gradiente de presión venosa hepática (GPVH), determinado por la diferencia de presión entre el territorio portal y las venas suprahepáticas. Este gradiente se encuentra aumentado en los pacientes cirróticos debido al bloqueo de las comunicaciones intersinusoidales secundario a la desestructuración del parénquima hepático. Se ha observado que, para que tenga lugar el sangrado, el GPVH debe ser mayor de $12 \mathrm{mmHg}$. Por lo tanto, gradientes inferiores al valor establecido como dintel permiten eliminar el riesgo de recidiva hemorrágica (4).

Ante unas cifras tan elevadas de mortalidad y recidiva, la profilaxis del sangrado está claramente indicada. Los métodos de los que actualmente se dispone para evitar el resangrado son las medidas farmacológicas combinadas o no con las técnicas endoscópicas.

Los betabloqueantes no cardioselectivos como el propranolol y el nadolol han demostrado ser eficaces en la prevención de la hemorragia (5) y la asociación con 5-mononitrato de isosorbide consigue reducciones de la presión portal mayores que los betabloqueantes solos (6).

La ligadura con bandas elásticas es hoy el tratamiento endoscópico de elección, ya que ha demostrado reducir significativamente la recidiva hemorrágica (7). La combinación de ambos métodos de tratamiento (farmacológico y endoscópico) reduce más el riesgo de sangrado que la ligadura endoscópica sola (8); aunque la utilidad del tratamiento combinado no está aún bien establecida.

En general, la decisión de elegir un tratamiento u otro se basa fundamentalmente en criterios endoscópicos; de manera que aquellos pacientes que presentaban varices pequeñas recibían tratamiento farmacológico y los pacientes con varices más grandes (grado III o IV) se sometían a la ligadura endoscópica con bandas elásticas. Sin embargo, la realización de un estudio hemodinámico en el que se valore la respuesta del GPVH antes y después de la administración de propranolol permite diferenciar a los pacientes que se beneficiarán de él de los que no lo harán; de esta forma se consigue optimizar el tratamiento y prescindir de fármacos innecesarios en pacientes que no van a responder al betabloqueante. Por ello, se ha recomendado la utilización de la respuesta del GPVH al tratamiento farmacológico como guía del tratamiento profiláctico (9), aunque en nuestro país no se dispone de información sobre el coste-efectividad de este procedimiento.

\section{OBJETIVO}

El objetivo del presente trabajo es determinar el costeefectividad de la realización del estudio hemodinámico previo a la elección del tratamiento de profilaxis secundaria de la hemorragia digestiva varicosa en pacientes cirróticos.

\section{MATERIAL Y MÉTODOS}

Para la realización de este estudio se consideraron dos grupos de enfermos. Grupo 1 (Tabla I): constituido por pacientes cirróticos de nuestro hospital (Hospital General Universitario de Alicante) que habían ingresado por un episodio de hemorragia digestiva varicosa. A todos los integrantes del grupo 1 se les realizó un estudio hemodinámico, previo a la elección del tratamiento profiláctico para valorar si los pacientes eran o no "respondedores" a propranolol. El estudio se realizó tras la suspensión del tratamiento con somatostatina, aproximadamente cinco días después del ingreso. La técnica, realizada con anes-

Tabla I. Grupo 1. Variables epidemiológicas

\begin{tabular}{lc}
\hline Edad & $56,6 \pm 9,2$ \\
Sexo $(H / M)$ & $24 / 12$ \\
Etiología cirrosis alcohólica/no alcohólica & $18 / 18$ \\
Child-Pugh & $8,1 \pm 2$ \\
Ascitis & 17 \\
Bilirrubina total (mg/dl) & $1,9(1-3,4)$ \\
Índice de Quick (\%) & $62,6 \pm 15,9$ \\
Plaquetas & $98.022 \pm 49.501$ \\
Tamaño varices I-II/III-IV & $13 / 23$ \\
Varices esofagogástricas & 9 \\
Seguimiento & $14,9 \pm 9,3$ \\
\hline
\end{tabular}

tesia local y condiciones de asepsia, consiste en la introducción de un catéter-balón a través de la vena yugular interna derecha, femoral o la cubital anterior hasta la vena hepática, donde, con el balón deshinchado, se registra la presión venosa libre. Posteriormente, tras hinchar el balón, se registra la presión venosa enclavada. El GPVH queda determinado por la diferencia entre ambas presiones, enclavada y libre (10). Tras un registro basal de presiones, se procede a la administración de propranolol intravenoso, a dosis de $0,15 \mathrm{mg} / \mathrm{kg}$; posteriormente y de la misma forma que se ha indicado, se determina el GPVH. Si este ha disminuido un $20 \%$ respecto al valor inicial o es menor a $12 \mathrm{mmHg}$, los pacientes con considerados "respondedores" y reciben tratamiento con betabloqueantes no cardioselectivos (11). A los pacientes "no respondedores" se les realiza ligadura endoscópica mediante bandas elásticas hasta erradicación de las varices. Según el diseño descrito por De Madaria y cols. (12).

Grupo 2: en los que la elección del tratamiento profiláctico se basó en criterios endoscópicos se modelizó a 
partir de los datos de la literatura (13). Así los pacientes con varices pequeñas recibieron tratamiento con propranolol combinado con nitratos y a los pacientes con varices grado III o IV se les realizó ligadura mediante bandas y además se les administró propranolol.

\section{Análisis coste-efectividad}

\section{Estrategias y modelo}

Elaboramos un árbol de decisión para evaluar el costeefectividad de las dos estrategias clínicas en el tratamiento de la hemorragia digestiva alta del paciente cirrótico: usando el GPVH o el tamaño de las varices para decidir la estrategia terapéutica (Fig. 1). Al comienzo del árbol de decisión se supone que los pacientes cirróticos presentan una HDA por varices esofágicas sin otras comorbilidades. En el modelo los pacientes pueden someterse a un estudio hemodinámico para la determinación del GPVH $\mathrm{y}$ ser etiquetados en respondedores o no respondedores que posteriormente pueden sufrir o no una recidiva del sangrado. En este brazo del árbol las probabilidades en cada rama corresponden a los hallazgos en un estudio prospectivo de cohortes realizado en nuestro hospital (12). Los pacientes no sujetos a estudio hemodinámico fueron sometidos a una endoscopia diagnóstico-terapéutica y clasificados de acuerdo al tamaño de las varices esofágicas pudiendo posteriormente sufrir o no una recidiva del sangrado. En este brazo del árbol las probabilidades en cada rama corresponden a datos de la literatura (13). Se asumió en el modelo que ninguno de los pacientes sometidos a estudio hemodinámico fue clasificado de

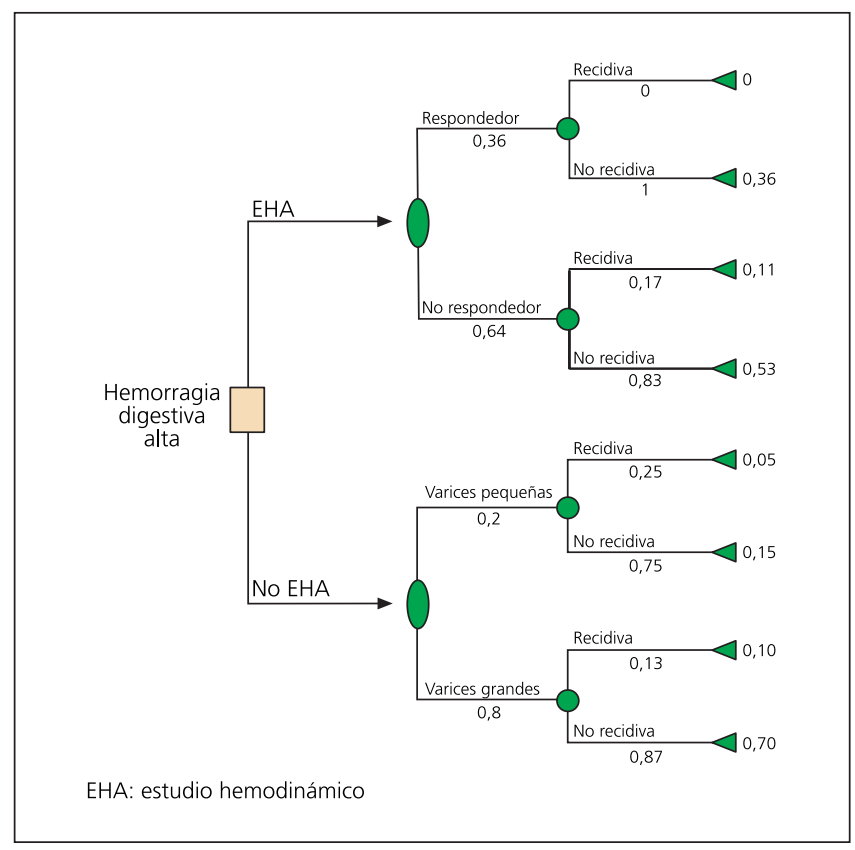

Fig. 1. acuerdo al tamaño de las varices ni viceversa. En todos los casos se consideró que la recidiva correspondía a una nueva HDA por varices esofágicas, que todos los pacientes pudieron clasificarse y que la tasa de abandonos a lo largo del estudio era similar en ambos brazos. Elegimos una duración del ciclo de 1 año para nuestro modelo con un máximo de una recidiva por paciente tras la cual saldría del estudio.

\section{Costes}

Los costes utilizados fueron obtenidos de la base de datos de costes sanitarios del Servicio de Información Económica del centro correspondientes al año 2004, coincidente en el tiempo con la inclusión de pacientes en el estudio prospectivo de cohortes realizado en nuestro hospital (12). Se tuvieron en cuenta los costes de gastroscopia, estudio hemodinámico, día de ingreso en Unidad de Sangrantes, sesión de tratamiento endoscópico con bandas y fármacos (costes de mercado de 2004 en España). En todos los pacientes se asumió un mismo tiempo de permanencia en la Unidad de Sangrantes (tiempo medio en nuestra experiencia de 5 días). Igualmente se asumió en todos los pacientes la realización de una gastroscopia diagnóstica al principio del estudio y en un $25 \%$ de estas gastroscopias diagnósticas la realización de un procedimiento terapéutico (colocación de bandas). En los pacientes tratados con bandas se asumió un tratamiento consistente en 3 sesiones incluyendo cada una de ellas el coste de una gastroscopia y de 1 kit de bandas. Para el cálculo de los costes se asumió una dosis diaria media de propranolol de $120 \mathrm{mg}$ /día. El resto de costes derivados del proceso asistencial de los pacientes (consultas externas, comorbilidades, etc.) se asumieron no diferentes en ambos grupos de estudio.

En el modelo no se consideraron costes indirectos ni intangibles. Todos los costes fueron calculados en euros. Al considerar un horizonte de 1 año y coincidir en el tiempo pacientes y costes no se consideró la aplicación de tasa de descuento alguna. Todos los análisis se realizaron desde la perspectiva del Sistema de Salud. Los costes empleados se resumen en el tabla II. Se calculó el coste incremental como la diferencia de costes de la estrategia que incluye la realización del EHD y la que no la incluye.

\section{Análisis de la efectividad}

La efectividad se expresó como frecuencias relativas de pacientes libres de resangrado al final del año de seguimiento. Se calculó la efectividad incremental como la diferencia de frecuencia relativa de resangrado de los pacientes sometidos a EHD y los clasificados de acuerdo al tamaño de las varices. Para cada estrategia se calculó una razón coste-efectividad dividiendo el coste de cada estrategia por la tasa de pacientes sin sangrado al final del se- 
guimiento obteniendo el coste por paciente sin sangrado al año. Se calculó el coste-efectividad incremental (CEI) como:

$$
\mathrm{CEI}=(\operatorname{coste} \mathrm{A}-\operatorname{coste} \mathrm{B}) /(\text { efectividad } \mathrm{A}-\text { efectividad } \mathrm{B})
$$

Siendo A la estrategia más efectiva para que el denominador sea positivo.

Los límites inferior y superior de los intervalos de confianza de las frecuencias relativas se calcularon usando la distribución binomial (14).

\section{Tabla II. Costes unitarios}

\begin{tabular}{lc}
\hline Coste unitario & Euros \\
\hline Día de ingreso en Unidad de Sangrantes & $2.234,12 €$ \\
Gastroscopia ( 1 kit de bandas en el 25\% de gastroscopias diagnósticas) & $142,47+95,48=237,95 €$ \\
Tratamiento endoscópico con bandas (3 gastroscopias + 3 kits de bandas) & $1.573,17 €$ \\
Estudio hemodinámico & $221,78 €$ \\
Propranolol (coste/año de tratamiento) & $69,64 €$ \\
\hline
\end{tabular}

Los costes se calcularon a partir del Servicio de Información Económica del Hospital General Universitario de Alicante correspondientes al año 2004. El coste de los fármacos se calculó a partir de los precios de mercado en el año 2004.

\section{RESULTADOS}

En el grupo 1, el porcentaje de "respondedores" a propranolol fue del $36 \%$. Y la tasa de resangrado de estos, observada en un año, del 0\%. De los "no respondedores", volvió a presentar hemorragia por varices un $17 \%$.

Atendiendo al grupo 2, observamos que la frecuencia de sangrado por varices grado I-II fue del $20 \%$. Estos pacientes recibieron profilaxis con propranolol y la tasa de recidiva al año fue del $28 \%$. La tasa de hemorragia por varices grandes (grado III o IV) fue del $80 \%$. A estos pacientes se les realizó ligadura endoscópica y además recibieron tratamiento con propranolol. La frecuencia de resangrado en este grupo fue de un $13 \%$.

\section{Resultados de costes}

La realización del EHD incrementa en 221,78 euros el coste del tratamiento de cada paciente (de 11.408,55 a 11.630,33 euros) antes de iniciar la estrategia terapéutica profiláctica. Este incremento representa apenas un 19,6\% del coste de atender el episodio de sangrado durante 5 días. Considerando el coste total al final del año, la opción terapéutica de menor coste es la de los pacientes no sometidos a EHD, con varices esofágicas pequeñas que son tratados con propranolol (11.478,19 euros). La siguiente opción de menor coste la representan los pacientes sometidos a un EHD que se muestran respondedores y son tratados sólo con propranolol (11.699,97 euros). Tanto los pacientes con varices grandes como los etiquetados como no respondedores en el EHD representaron un cos- te más elevado a expensas del tratamiento endoscópico con bandas (13.337,80 y 13.489,94 euros, respectivamente). Estos costes se incrementan en los pacientes que sufren un nuevo episodio de sangrado (Tabla III).

En la tabla III se aprecia que, a pesar de que el tratamiento tanto de los pacientes respondedores como de los no respondedores según el EHD es más costoso que el de los pacientes clasificados según el tamaño de las varices, cuando este coste se considera en función del porcentaje esperado de recidivas y no recidivas el incremento medio del coste de no realizar el EHD es de 576,67 euros, aunque el intervalo de posibles valores es muy amplio, oscilando desde un ahorro al realizar el EHD de 3.478,51 euros hasta un posible incremento del gasto de hasta $3.580,11$ euros, dependiendo de las frecuencias de recidiva que puedan darse en cada uno de los grupos analizados.

\section{Resultados de efectividad}

En la figura 1 se indican las probabilidades esperadas (en tanto por uno) en cada uno de los brazos considerados. Como se ha indicado previamente, los datos correspondientes a los pacientes sometidos a un EHD se han tomado de un estudio prospectivo realizado en nuestro servicio. En este estudio un $36 \%$ de los pacientes estudiados fue respondedor $(n=9)$ y ninguno de ellos resangró en el siguiente año (frecuencia relativa de 0,00 ; IC95\%: $0,00-0,34)$. Entre los no respondedores $(64 \%$ de pacientes estudiados; $\mathrm{n}=17$ ) la tasa de resangrado fue de 0,17 (IC95\%: 0,03-0,38). En el grupo de los pacientes tratados de acuerdo al tamaño de las varices (datos de la literatura) la frecuencia de resangrado en aquellos con varices pequeñas tratados con la combinación de propranolol y nitratos es de 0,25 (IC95\%: 0,17-0,35) mientras que en pacientes con varices grandes tratados tanto con fármacos como con bandas la frecuencia de resangrado es de 0,13 (IC95\%: 0,08-0,21).

En conjunto se aprecia una frecuencia de recidiva anual de sangrado de 0,11 (IC95\%: 0,02-0,27) en el grupo de pacientes clasificado de acuerdo con el EHD y de 0,16 (IC95\%: 0,09-0,25) en el grupo de pacientes tratados según el tamaño de las varices. La efectividad del EHD o tasa de pacientes libres de resangrado al año sería de 0,89 (IC95\%: 0,64-0,98) y la de la estrategia consistente en clasificar a los pacientes por el tamaño de las varices sería de 0,84 (IC95\%: 0,75-0,90).

\section{Razón coste-efectividad}

La razón coste-efectividad para el EHD es de 15.843,25 (IC95\%: 9.777,46-23.960,01) euros/paciente libre de resangrado al año de seguimiento. Para la estrategia basada en el tamaño de las varices la razón coste-efectividad es de 17.267,25 (IC95\%: 14.329,94-20.875,65) euros/paciente libre de resangrado al año de seguimiento. 
Tabla III.

\begin{tabular}{|c|c|c|c|c|}
\hline$E H D$ & Frecuencia & $\begin{array}{l}\text { Coste } \\
\text { (euros) }\end{array}$ & Coste-frecuencia & $\begin{array}{l}\text { Coste-frecuencia } \\
\text { incremental }\end{array}$ \\
\hline Respondedores & 0,36 & & & \\
\hline HDA inicial + tto. farmacológico + recidiva & $\begin{array}{c}0,00 \\
(0,00-0,12)\end{array}$ & $23.108,52$ & $\begin{array}{c}0,00 \\
(0,00-2.773,02)\end{array}$ & \\
\hline HDA inicial + tto. farmacológico & $\begin{array}{c}0,36 \\
(0,24-0,36)\end{array}$ & $11.699,97$ & $\begin{array}{c}4.211,99 \\
(2.807,99-4.211,99)\end{array}$ & \\
\hline No respondedores & 0,64 & & & \\
\hline HDA inicial + bandas + recidiva & $\begin{array}{c}0,11 \\
(0,02-0,24)\end{array}$ & $24.898,49$ & $\begin{array}{c}2.738,83 \\
(497,97-5.975,64)\end{array}$ & \\
\hline HDA inicial + bandas & $\begin{array}{c}0,53 \\
(0,40-0,62)\end{array}$ & $13.489,94$ & $\begin{array}{c}7.149,67 \\
(5.395,98-8.363,76)\end{array}$ & \\
\hline Total EHD & 1,00 & & $\begin{array}{c}14.100,49 \\
(8.701,94-21.324,41)\end{array}$ & \\
\hline $\begin{array}{l}\text { Coste incremental de realizar el EHD vs. } \\
\text { clasificar los pacientes por tamaño de varices }\end{array}$ & & & & $\begin{array}{c}-576,67 \\
(-3.478,51-3.580,11)\end{array}$ \\
\hline \multicolumn{5}{|l|}{ No EHD } \\
\hline Varices pequeñas & 0,20 & & & \\
\hline HDA inicial + tto. farmacológico + recidiva & $\begin{array}{c}0,05 \\
(0,04-0,08)\end{array}$ & $22.886,74$ & $\begin{array}{c}1.144,34 \\
(915,47-1.830,94)\end{array}$ & \\
\hline HDA inicial + tto. farmacológico & $\begin{array}{c}0,15 \\
(0,12-0,16)\end{array}$ & $11.478,19$ & $\begin{array}{c}1.721,73 \\
(1.377,38-1.836,51)\end{array}$ & \\
\hline Varices grandes & 0,80 & & & \\
\hline HDA inicial + tto. farmacológico + bandas + recidiva & $\begin{array}{c}0,10 \\
(0,06-0,17)\end{array}$ & $24.746,35$ & $\begin{array}{c}2.474,64 \\
(1.484,78-4.206,88)\end{array}$ & \\
\hline HDA inicial + tto. farmacológico + bandas & $\begin{array}{c}0,70 \\
(0,63-0,74)\end{array}$ & $13.337,80$ & $\begin{array}{c}9.336,46 \\
(8.402,82-9.869,97)\end{array}$ & \\
\hline Total No EHD & 1,00 & & $\begin{array}{c}14.677,16 \\
(12.180,45-17.744,30)\end{array}$ & \\
\hline
\end{tabular}

Entre paréntesis se indica el IC 95\% (límite inferior-límite superior). HDA: hemorragia digestiva alta; tto.: tratamiento. Todos los costes corresponden a euros.

El coste-efectividad incremental es de un ahorro de $14.416,76$ euros por cada paciente adicional que no resangra en un año gracias al EHD.

\section{Análisis de sensibilidad}

Se realizó un análisis de sensibilidad variando el porcentaje de pacientes respondedores en el EHD, manteniendo fijo el porcentaje de pacientes con varices pequeñas en un $20 \%$ y modificando el porcentaje de pacientes con varices pequeñas manteniendo fijo el porcentaje de respondedores en el EHD en un 36\%. En la figura 2 se aprecia que la variación del porcentaje de pacientes con varices pequeñas varía muy poco el coste incremental comparado con la realización del EHD, sin embargo sí influye de manera notable en el resultado económico el porcentaje de respondedores en el EHD, de manera que si este es inferior al 20\%, la estrategia que incluye el EHD es menos coste-efectiva que la clasificación de los pacientes atendiendo sólo al tamaño de las varices. La estrategia basada en el EHD es coste-efectiva cuando en la población estudiada el número de respondedores es mayor al 20\%. Igualmente, se realizó un análisis en el que, asumiendo fijas las tasas de respondedores y no respondedores del EHD y las tasas de varices pequeñas y grandes es-

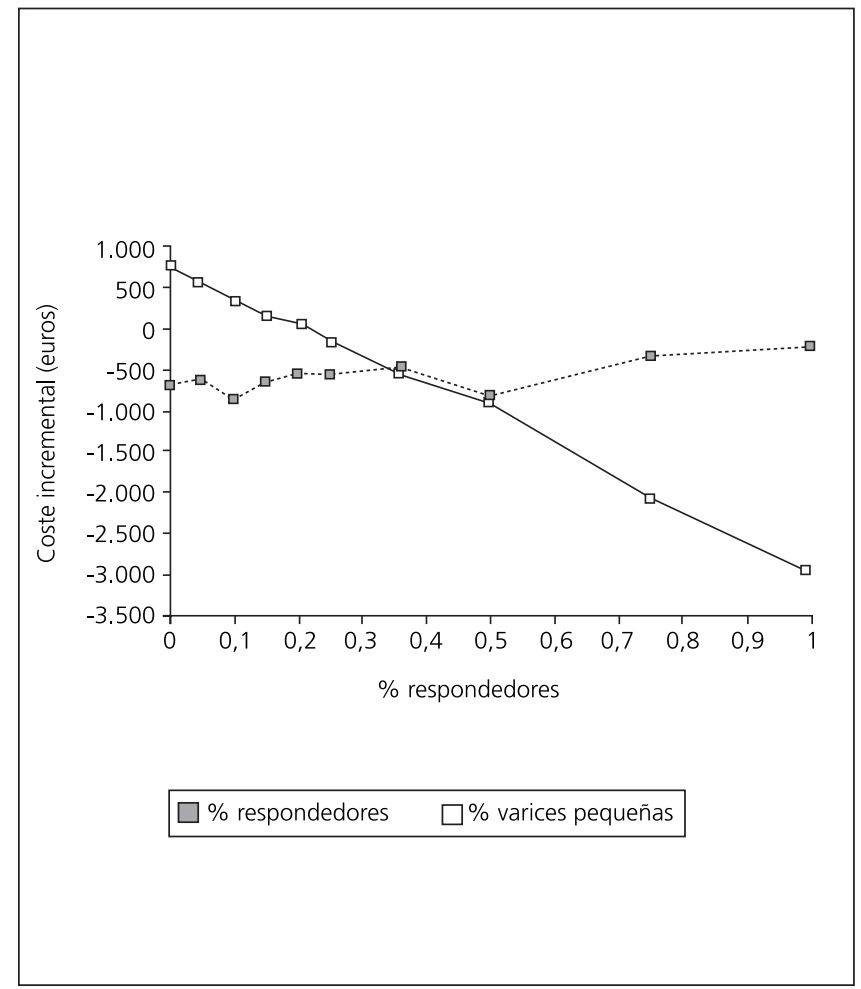

Fig. 2. 
tablecidas en el modelo inicial, se variaron las tasas de recidiva de cada uno de estos grupos (Fig. 3). En este caso se aprecia que el factor importante a la hora de considerar la relación coste-efectividad del EHD es la tasa de resangrado en los pacientes respondedores y no respondedores clasificados de acuerdo a este método diagnóstico. Es necesario que las tasas de resangrado en los grupos de pacientes con varices pequeñas sean del $0 \%$ o inferiores al $5 \%$ en el caso de las varices grandes para que la estrategia del EHD no resulte coste-efectiva. Por otro lado, el EHD deja de ser coste-efectivo si las tasas de resangrado en el grupo de respondedores son mayores al $15 \%$ y en el grupo de no respondedores superan el 25\%, límites, ambos, superiores a los valores máximos del IC $95 \%$ calculados a partir de nuestra experiencia.

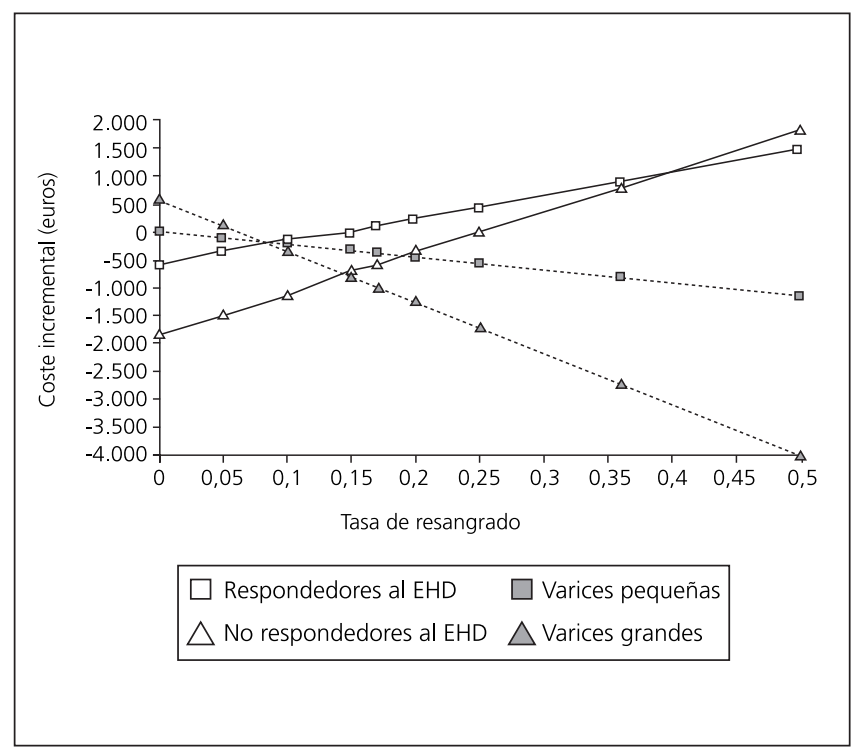

Fig. 3.

\section{DISCUSIÓN}

Según los resultados obtenidos en el presente estudio, la monitorización del gradiente de presión venosa hepática previa a la elección de la profilaxis de la hemorragia digestiva varicosa se presenta como un procedimiento útil. En nuestro trabajo, la medición del gradiente combinado con el tratamiento médico y/o endoscópico puede reducir los costes hasta en 576,67 $€$. El coste-efectividad de la monitorización del GPVH para guiar la profilaxis de la hemorragia varicosa depende en gran medida de los costes de la medición del gradiente, la esperanza de vida y la tasa de resangrado (15).

En el trabajo de Hicken y cols. (16) la medición del GPVH es una estrategia cara para reducir la hemorragia varicosa o la mortalidad, especialmente en pacientes con una esperanza de vida corta. Si atendemos al trabajo de
Imperiale y cols. (17) los resultados del coste-efectividad de la medición del GPVH son variables en función del análisis de sensibilidad. Según nuestros resultados, la estrategia basada en el EHD es coste-efectiva cuando en la población estudiada el número de respondedores es mayor al $20 \%$. Por otro lado, el EHD deja de ser coste-efectivo si las tasas de resangrado en el grupo de respondedores son mayores al $15 \%$ y en el grupo de no respondedores superan el 25\%, límites, ambos, superiores a los valores máximos del IC $95 \%$ calculados a partir de nuestra experiencia.

Así mismo, el tratamiento tras la realización del estudio hemodinámico demuestra disminuir la frecuencia de recidiva anual de sangrado, 0,11 (IC 95\%: 0,02-0,24) en el grupo al que se le ha clasificado según el estudio, frente a 0,15 (IC 95\%: 0,08-0,25), en el grupo de pacientes tratados en función del tamaño de las varices. Esto puede ser atribuido a que la medición del gradiente permite monitorizar la respuesta al tratamiento y optimizar el mismo en función de esta.

De todos nuestros pacientes "respondedores" $(\mathrm{n}=9)$ tratados con propranolol, ninguno resangró en el año siguiente (frecuencia relativa de 0,00). La explicación de esto puede encontrarse en varios factores; el primero de ellos es que la mayoría de los incluidos presentaban hepatopatía en estadio A-B de Child-Pugh $(8,1 \pm 2)$, lo que explica la menor tasa de complicaciones que acontece en estos pacientes. Y el segundo es el periodo de seguimiento relativamente corto, lo cual podría justificar que no se observaran resangrados, en contra de lo que cabría esperar si este plazo se ampliara. Hemos considerado interesante la realización de este estudio, ya que en nuestro país no se dispone de datos que permitan establecer el costeefectividad de introducir este procedimiento en la práctica clínica (18-21). Los costes que hemos utilizado son los que calcula nuestro centro (Hospital General Universitario de Alicante). Estos no tienen por qué coincidir con los costes en un mercado libre, pero sí son representativos de nuestro sistema de salud.

Por otra parte, la disponibilidad de marcadores hemodinámicos permite optimizar el tratamiento profiláctico de la hemorragia por varices esofagogástricas, evitando la utilización de betabloqueantes en pacientes que no se benefician de ellos y los efectos secundarios que de su uso pueden derivar.

En conclusión, creemos que en nuestro medio la realización del estudio hemodinámico se muestra como una herramienta coste-efectiva en la profilaxis de la hemorragia digestiva varicosa en pacientes cirróticos y mantiene una relación coste-efectiva favorable, comparado con la no realización del estudio mientras la tasa de recidiva de la hemorragia, en los que se realiza el estudio, sea inferior al $20 \%$ y mientras que supere el $5 \%$ en los que no se realiza. Además, la disponibilidad de marcadores hemodinámicas permite optimizar el tratamiento profiláctico y prescindir medidas terapéuticas en pacientes que no se beneficien de ellas. 


\section{BIBLIOGRAFÍA}

1. The North Italian Endoscopic Club for the Study and Treatment of Esophageal Varices. Prediction of the first variceal hemorrage in patients with cirrhosis of the liver and esophageal varices: A prospective multicenter study. N Engl J Med 1988; 319: 983-9.

2. Groszmann RJ, Bosch J, Grace ND, Conn HO, García-Tsao G, Navasa $\mathrm{M}$, et al. Hemodinamic events in a prospective randomized trial of propranolol versus placebo in the prevention of a firs variceal hemorrhage. Gastroenterology 1990; 99: 1401-7.

3. Bosch J, García-Pagán JC. Prevention of variceal rebleeding. Lancet 2003; 361: 952-4.

4. García-Tsao G, Grozzman RJ, Fisher R, et al. Portal pressure, presence of gastroesophageal varices and variceal bleeding. Hepatology 1985; 5: 419-24.

5. Bernard B, Lebrec D, Mathurin P, Opolon P, Poynard T. Beta-adrenergic antagonists in the prevention of gastrointestinal rebleeding in patients with cirrosis: A metaanalysis. Hepatology 1997; 25: 63-70.

6. García-Pagán JC, Feu F, Bosch J, Rodés J. Propranolol compared with propranolol plus isosorbide-5-mononitrate for portal hypertension in cirrhosis. A randomized controlled study. Ann Intern Med 1991; 114: 869-73.

7. De Francis R, Primignani M. Endoscopic treatments for portal hypertension. Semin Liver Dis 1999; 19: 439-55.

8. Lo GH, Lai KH, Cheng JS, Chen MH, Harar MC, Hsu PI. Endoscopic variceal ligation plus nadolol and sucralfate compared with ligation alone for the prevention of variceal rebleeding: A prospective, randomized trial. Hepatology 2000; 32: 461-5.

9. Abraldes JG, Tarantino I, Turnes J, García-Pagán JC, Rodes J, Bosch J. Hemodynamic response to pharmacological treatment portal hypertension and long-term prognosis of cirrhosis. Hepatology 2003; 37: 902-8.

10. Bosch J, García-Pagán JC, Berzigotti A, Abraldes JG. Measurement of portal pressure and its role in the management of chronic liver dis- ease. Semin Liver Dis 2006; 26 (4): 348-62.

11. Feu F, et al. Relation between portal pressure response to pharmacological therapy and risk of recurrent variceal hemorrhage in patients with cirrhosis. Lancet 1995; 346: 1056-9.

12. De Madaria E, Palazón JM, Sánchez J, de España F, Gil S, Pascual S, et al. Monitoring of HVPG during pharmacological therapy: A comparison between two methods. J Hepatol 2005; 42 (Supl. 2): 79.

13. Sharara AI, Jockey DC. Gastroesophageal variceal hemorrhage. N Engl J Med 2001; 345: 669-81.

14. Carrasco JL. El método estadístico en la investigación médica. $6^{\mathrm{a}}$ ed. Madrid: Editorial Ciencia 3; 1995.

15. Raines DL, Dupont AW, Arguedas MR. Cost-effectiveness of hepatic venous pressure gradient measurements for prophylaxis of varicela re-bleeding. Aliment Pharmacol Ther 2004; 19 (5): 571-81.

16. Hicken BL, Sharara AI, Abrams GA, Eloubeidi M, et al. Hepatic venous pressure gradient measurements to assess response to primary prophylaxis in patients with cirrhosis: A decision analytical study. Aliment Pharmacol Ther 2003; 17 (1): 145-53.

17. Imperiale TF, Chalasani N, Klein RW. Measuring the hemodynamic response to primary pharmacoprophylaxis of variceal bleeding: A costeffectiveness analysis. Am J Gastroenterol 2003; 98 (12): 2742-50.

18. Spiegel BM, Targownic L, Dulai GS, Karsan HA, et al. Endoscopic screening for esophageal varices in cirrhosis. Is it ever cost effctive? Hepatology 2003; 37 (2): 366-7.

19. Targownic L, Spiegel B, Dulai GS, Karsan HA, et al. The cost-effectiveness of hepatic venous pressure gradient monitoring in the prevention of recurrent variceal hemorrhage. Am J Gastroenterol 2004; 99 (7): 1306-15.

20. Rubenstein JH, Eisen GM, Inadomi JM. A cost-utility analysis of secondary prophylaxis for variceal hemorrhage. Am J Gastroenterol 2004; 99 (7): 1274-88.

21. Saab S, De Rosa V, Nieto J, Durazo F, et al. Costs and clinical outcomes of primary prophylaxis of variceal bleeding in patients with hepatic cirrhosis: A decision analytic model. Am J Gastroenterol 2003; 98 (4): 763-70. 